\title{
THE STRENGTH OF LOWER LIMBS IN ELITE GYMNASTS AND SWIMMMERS
}

\author{
Aleksander Wiażewicz, A, B, C, D Waldemar Wiśniowski, 2, A, B, D Tomasz Sacewicz, 2, A, B, D \\ Magdalena Czajka ${ }^{3, A, B, D}$
}

\author{
${ }^{1}$ University of Szczecin, Faculty of Health and Physical Education, Poland \\ 2 Józef Piłsudski University of Physical Education, Faculty of Physical Education and Sport, Biała Podlaska, Poland \\ ${ }^{3}$ Józef Piłsudski University of Physical Education, Student of the Faculty of Physical Education and Sport, Biała Podlaska, Poland

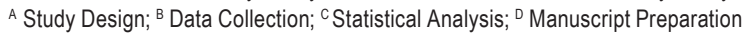

\author{
Address for correspondence: \\ Aleksander Wiażewicz \\ Faculty of Health and Physical Education, the University of Szczecin \\ Al. Piastów 40b, blok 6, 71-065 Szczecin, Poland \\ E-mail: Aleksander.Wiazewicz@usz.edu.pl
}

\begin{abstract}
Ahstract Critical moments during gymnastic exercises are those related to landing and suppressing overloads, transmitted through lower limbs. A swimmer's lower limbs provide a good body position in the water and maintain the balance. Both in sports authors postulate reaching bilateral, steady development of the athlete's body.

The main aim was identification and a comparative analysis of the hip, knee and ankle joints flexors and extensors strength, of highly qualified gymnasts and swimmers.

Ten healthy subjects participated in the investigation. Five gymnasts $(22.2 \pm 7.3$ y.0., $169.4 \pm 3.91 \mathrm{~cm}, 64.4 \pm 3.78 \mathrm{~kg})$ and five swimmers (20.5 \pm 1.3 y.0., $186.2 \pm 1.64 \mathrm{~cm}, 78.4 \pm 2.61 \mathrm{~kg}$ ) were highly qualified Polish athletes.

Strength measurements were made on Biodex S4 isokinetic dynamometer. Subjects performed hip extension/flexion in lying position, knee extension/flexion and ankle plantar/dorsi flexion.

Strength parameters of the lower limbs differentiate gymnasts and swimmers.

Lower limb's strength parameters can be used during training, control, selection or prevention. To achieve a high sport level, swimmers must demonstrate higher strength parameters of lower limbs than gymnasts. The value of work in the maximum repetition (MRW) should be thoroughly analyzed. Highly qualified gymnasts and swimmers should take into account the harmonious, bilateral strength development of the lower limbs.
\end{abstract}

Key WOrdls lower limbs, strength, Biodex, gymnasts, swimmers

\section{Introduction}

Sport performance is a special manifestation of human functioning during which particular body systems often work in conditions of maximum potential. Even the hardest professional work does not cause in the human body such adaptive changes that occur in master class athletes (Bołoban, Wiśniowski, Mistułowa, Niźnikowski, 2003; Sadowski, Bołoban, Mastalerz, Niźnikowsk, 2003). Muscle strength affects all aspects of human activity. It is treated as a measure and index of health. The development of muscular strength and keeping it at a constant high 
level is of great importance in sport, correction and rehabilitation. It is the basis of all forms of physical activity and a parameter that determines the development of other motor abilities (Jensen, Smith, Johnson, 1971; Wit, 2002).

Gymnasts are exposed to large overloads of the musculoskeletal and articular system, which often leads to injury. In the case of sport gymnasts, the whole skeletal system is exposed to injuries due to the specificity of the discipline (six events requiring a performer to move in space, perform complex elements and landings). The gymnasts' arms are most often exposed to symmetrical, bilateral loads added to the upper limbs (Gerhardt, Doyscher, Boschert, Scheibel, 2014). However, trainers shouldn't play down the role of lower limbs. The critical moments during the exercises are those related to landing and suppressing overloads. Ground reactions exceed $10,000 \mathrm{~N}$ and are transmitted through the ankle joint to the knee and hip joints (Bober, Hay, 1991; Kędzior, Niwiński, Wit, 1992; Czajka, Wiśniowski, Sacewicz, 2016). Incorrect performance of the exercise, caused by bad technique or musculoskeletal system dysfunction, may result in mechanical damage to the joints.

Swimmers and gymnasts train in artificially created conditions (swimming pool - water environment, gymnastic devices). The position of a swimmer is unusual for a human - horizontal, and the work done in the training process is in a different environment than gymnasts (constant water resistance, constant movement). The speed of swimming depends on many factors. As one of the most important, the strength abilities of a swimmer are mentioned. While training in a swimming pool, a swimmer stays in motion all the time, which significantly strains the musculoskeletal system. The basic drag force of athletes practicing sports swimming is generated by the arms (Troszczyński, 1999; Przybylska, 2010). Nevertheless, many authors (Bogajewski, Roszko, Witkowski, Wróbel, 1969; Bartkowiak, 1972, 1984; Troszczyński, 1999; Przybylska, 2010) report that the proper functioning of the lower limbs provides a good body position in the water and maintains the balance. The work of the lower limbs is as important as the upper limbs, although the legs do not give such a large propelling force (Bartkowiak, 1984; Laughlin, 2007; Montgomery, Chambers, 2009). The most common injuries occurring in swimming include various permanent and chronic upper limb injuries: bruises, wounds, painful shoulder syndrome (functional instability of the glenohumeral joint), rotator cuff injury, bicep arm inflammation, shoulder dislocation. However, there are also injuries of the lower limbs, i.e. the "swimmer's knee" or pain syndrome of the patellofemoral joint (Czabański, Fiłon, Zatoń, 2003).

Both in swimming (Alonso-Cortés Fradejas et al., 2006; Wiażewicz 2015; Wiażewicz, 2016) and gymnastics (Jurkojć, Michnik, Skubacz, Ziółkowska, 2012; Gerhardt et al., 2014) authors postulate reaching bilateral steady development of the athlete's body. It is reported that deficits in strength between body sides and the agonists to antagonists strength ratio are important in injury prevention of these athletes. Improper proportions in strength between the right and left sides or between particular muscle groups can result in poor posture during gymnastic events and inadequate stabilization of each joint or the entire body. This may result in micro-injuries as well as more frequent trauma (Jurkojć et al., 2012). This may also lead to unwanted torso rotation, changes in swimming techniques and reduced mobility in the water (Sanders, 2013).

\section{Main aim}

The main aim of this study was identification of the hip, knee and ankle joint flexor and extensor strength, in highly qualified athletes practicing gymnastics and swimming. The next objective was a comparative analysis of the gymnasts' results with the results of the swimmers in each movement, in the examined joints. The results provided answers to the following research questions:

1. What values are the selected strength parameters in highly qualified gymnasts and swimmers? 
2. Do the results differentiate the examined groups?

3. Which parameter differentiates the examined groups the most?

\section{Methods}

Ten healthy subjects (five gymnasts and five swimmers) participated in the study. The gymnasts $(22.2$ \pm 7.3 years old, $169.4 \pm 3.91 \mathrm{~cm}, 64.4 \pm 3.78 \mathrm{~kg}$ ) were highly qualified Polish athletes. Two of them were Master of Sports International Class (MM), one was Master of Sports (M) and two were $1^{\text {st }}$ class (I). The participants were informed about the task and gave their written permission for the tests. The second group were swimmers (20.5 \pm 1.3 years old, $186.2 \pm 1.64 \mathrm{~cm}, 78.4 \pm 2.61 \mathrm{~kg}$ ). They were high class athletes from the City Swimming Club in Szczecin (MKP). Two subjects were Master of Sports (M), while three of them were Master of Sports International Class (MM). Participants were informed about the task and gave their written permission to the test. The Bioethics Committee at the Regional Medical Chamber in Szczecin gave a positive opinion about the research project (resolution No. 15/KB/V/2013 dated 10.12.2013 r.) - applies to the swimmers.

First, the subjects performed a 5 -minute warm up on a stationary bike, then a 5 -minute warm up focused on the investigated joint. Strength measurements were made on a Biodex S4 isokinetic dynamometer (Biodex Corp., Shirley, NY, USA). During the test, the subjects performed hip extension/flexion in a lying position, knee extension/ flexion and ankle plantar/dorsi flexion in two different protocols. The number of repetitions and angular velocity are presented in Table 1. There was a 2-minute break between protocols and a 4-minute rest between right and left limb measurement. Preparation and the measurement were made according to Biodex Medical Systems, Inc. (Biodex, 2019a). Each joint was tested on a different day.

Table 1. Number of repetitions and angular velocity in Protocols 1 and 2 in the tested joints

\begin{tabular}{llcccc}
\hline \multirow{2}{*}{ Joint } & \multirow{2}{*}{ Pattern } & \multicolumn{2}{c}{ Protocol 1 } & Protocol 2 \\
\cline { 3 - 6 } & & Repetitions & Angular velocity $\left({ }^{\circ} / \mathrm{s}\right)$ & Repetitions & Angular velocity $\left({ }^{\circ} / \mathrm{s}\right)$ \\
\hline Hip & Flexion/Extension & 5 & 45 & 10 & 300 \\
Knee ${ }^{*}$ & Extension/Flexion & 5 & 60 & 10 & 180 \\
Ankle & Plantar/Dorsi Flexion & 5 & 30 & 10 & 60 \\
\hline Gravity correction was applied. & & & &
\end{tabular}

Values: peak torque (PT), peak torque to body weight ratio (PT/BW) and maximal repetition work (MRW) were used from protocol 1. Values: work to body weight ratio (W/BW), total work (TW), work fatigue (WF), average power (AP), average peak torque (APT) and agonist to antagonist ratio (AG:AN) were taken from protocol 2. All were measured for both movements in the tested joints. In order to determine the asymmetry value, the percentage deficits of the selected strength parameters (PT, MRW, TW and AP) were analyzed.

For statistical analyses, Statistica v13.5 was used. A Shapiro-Wilk test (significance level 0.05) was used to check whether samples came from a normally distributed population. T-Test: two-sample assuming equal variances was used (significance level 0.05), when the result of Shapiro-Wilk's test wasn't significant in both compared variables, so samples came from a normally distributed population. Results were presented as mean $(\bar{x})$ and standard deviation (SD). Otherwise, when the result of Shapiro-Wilk's test was significant in at least one of the two compared variables, samples did not come from a normally distributed population and a nonparametric MannWhitney $U$ test was used. In this case the median and the quartile gap were presented. 


\section{Results}

Results from the hip joint investigation are presented in Table 2. Almost all average maximum strength parameters from protocol 1 (PT, PT/BW, MRW) measured in the hip joint movements were significantly higher for swimmers than gymnasts. Right hip extension movement presented almost three times significantly greater values for W/BW, TW and AP in swimmer than in gymnasts. In hip flexion APT was higher for gymnasts - significant difference only for the left joint. But the same parameter in extension was almost twice higher for swimmers, with significant differences only for the right joint. Differences between both groups in AG:AN ratio were significant. Average values were more than twice higher from the gymnasts.

Tahle 2. Comparison of gymnasts and swimmers hip strength parameters

\begin{tabular}{|c|c|c|c|c|c|c|c|}
\hline & & & \multirow{2}{*}{\multicolumn{2}{|c|}{ Gymnasts }} & & & \multirow{3}{*}{$p$} \\
\hline & & & & & \multicolumn{2}{|c|}{ Swimmers } & \\
\hline & & & $\bar{x}$ & SD & $\bar{x}$ & SD & \\
\hline \multirow{4}{*}{ PT } & \multirow{2}{*}{ Flexion } & Right (Nm) & 84.9 & 14.64 & 133.2 & 26.75 & $0.007587^{\star}$ \\
\hline & & Left (Nm) & 72.0 & 17.09 & 143.2 & 35.62 & $0.003808^{*}$ \\
\hline & \multirow{2}{*}{ Extension } & Right (Nm) & 98.4 & 14.81 & 206.0 & 55.33 & $0.002990^{*}$ \\
\hline & & Left (Nm) & 76.2 & 18.89 & 210.6 & 56.35 & $0.000984^{*}$ \\
\hline \multirow{4}{*}{ PT/BW } & \multirow{2}{*}{ Flexion } & Right (\%) & 132.1 & 21.58 & 171.6 & 29.43 & $0.041508^{*}$ \\
\hline & & Left (\%) & 111.6 & 23.20 & 185.1 & 39.93 & $0.007448^{*}$ \\
\hline & \multirow{2}{*}{ Extension } & Right (\%) & 152.8 & 19.72 & 266.0 & 62.57 & $0.004830^{*}$ \\
\hline & & Left $(\%)^{* *}$ & 112.2 & 25.6 & 288.4 & 25.50 & $0.007937^{\star}$ \\
\hline \multirow{4}{*}{ MRW } & \multirow{2}{*}{ Flexion } & Right (J) & 58.6 & 6.70 & 117.3 & 21.37 & $0.000378^{*}$ \\
\hline & & Left (J) & 42.9 & 9.30 & 113.3 & 5.31 & $0.000000^{*}$ \\
\hline & \multirow{2}{*}{ Extension } & Right (J) & 58.4 & 15.89 & 178.2 & 48.61 & $0.000787^{*}$ \\
\hline & & Left (J) & 43.0 & 14.38 & 178.5 & 49.72 & $0.000380^{*}$ \\
\hline \multirow{4}{*}{ W/BW } & \multirow{2}{*}{ Flexion } & Right (\%) & 40.5 & 17.59 & 40.1 & 16.48 & 0.967008 \\
\hline & & Left (\%) & 35.8 & 10.78 & 26.8 & 11.08 & 0.230133 \\
\hline & \multirow{2}{*}{ Extension } & Right (\%) & 41.9 & 14.25 & 108.8 & 53.96 & $0.027913^{*}$ \\
\hline & & Left (\%) & 38.7 & 11.64 & 93.6 & 61.71 & 0.086151 \\
\hline \multirow{4}{*}{ TW } & \multirow{2}{*}{ Flexion } & Right (J) & 215.6 & 124.66 & 237.1 & 114.63 & 0.784296 \\
\hline & & Left $(J)$ & 185.8 & 73.68 & 134.1 & 62.56 & 0.266274 \\
\hline & \multirow{2}{*}{ Extension } & Right (J) & 230.2 & 89.58 & 661.5 & 348.58 & $0.027932^{*}$ \\
\hline & & Left (J) & 208.3 & 70.38 & 550.2 & 364.97 & 0.073724 \\
\hline \multirow{4}{*}{ WF } & \multirow{2}{*}{ Flexion } & Right (\%) & -11.6 & 9.80 & 20.1 & 68.30 & 0.916815 \\
\hline & & Left (\%) & -28.9 & 37.17 & -30.7 & 83.40 & 0.967434 \\
\hline & \multirow{2}{*}{ Extension } & Right (\%) & -8.6 & 18.42 & -42.3 & 51.94 & 0.208949 \\
\hline & & Left (\%) & 1.1 & 30.52 & -37.5 & 53.75 & 0.199475 \\
\hline \multirow{4}{*}{ AP } & \multirow{2}{*}{ Flexion } & Right (W) & 58.5 & 42.13 & 61.8 & 35.20 & 0.895772 \\
\hline & & Left (W) & 51.6 & 26.84 & 35.4 & 17.26 & 0.288527 \\
\hline & \multirow{2}{*}{ Extension } & Right (W) & 69.1 & 35.96 & 198.8 & 115.76 & $0.043685^{*}$ \\
\hline & & Left (W) & 59.6 & 34.28 & 162.3 & 106.55 & 0.074162 \\
\hline & Flexion & Right (Nm) & 55.1 & 16.80 & 44.3 & 19.13 & 0.372306 \\
\hline APT & riexion & Left (Nm) & 47.9 & 11.51 & 25.2 & 8.55 & $0.007585^{*}$ \\
\hline API & Fytencion & Right (Nm) & 47.4 & 13.67 & 101.7 & 49.37 & $0.045058^{*}$ \\
\hline & Exlension & Left (Nm) & 45.1 & 17.00 & 95.7 & 54.84 & 0.084140 \\
\hline$A G \cdot A N$ & & Right (\%) & 116.3 & 38.28 & 49.6 & 11.80 & $0.005853^{*}$ \\
\hline AG:AN & & Left (\%) & 110.8 & 31.13 & 35.8 & 12.28 & $0.001034^{*}$ \\
\hline
\end{tabular}

"Difference between both groups is significant $(p<0.05)$.

"A nonparametric Mann-Whitney $\mathrm{U}$ test was used. Results are median and the quartile gap. 
Table 3 presents a comparison of the gymnasts and swimmers knee strength. Similarly to the hip joint, almost all PT, PT/BW, MRW values were significantly higher for the swimmers than gymnasts. In all protocol 2 parameters the differences between groups were non-significant. But almost every value was higher for the swimmers.

Table 3. Comparison of gymnasts and swimmers knee strength parameters

\begin{tabular}{|c|c|c|c|c|c|c|c|}
\hline & & & \multirow{2}{*}{\multicolumn{2}{|c|}{ Gymnasts }} & \multirow{2}{*}{\multicolumn{2}{|c|}{ Swimmers }} & \multirow{3}{*}{$P$} \\
\hline & & & & & & & \\
\hline & & & $\bar{x}$ & SD & $\bar{x}$ & SD & \\
\hline \multirow{4}{*}{ PT } & \multirow{2}{*}{ Extension } & Right (Nm) & 126.8 & 35.92 & 229.2 & 40.43 & 0.002855 \\
\hline & & Left (Nm) & 128.6 & 15.32 & 231.0 & 37.91 & 0.000509 \\
\hline & \multirow{2}{*}{ Flexion } & Right (Nm) & 72.3 & 11.10 & 119.5 & 19.55 & 0.001552 \\
\hline & & Left (Nm) & 69.8 & 12.33 & 120.4 & 8.67 & $0.000069^{\circ}$ \\
\hline \multirow{4}{*}{ PT/BW } & \multirow{2}{*}{ Extension } & Right (\%) & 196.4 & 49.02 & 302.1 & 49.90 & 0.009658 \\
\hline & & Left (\%) & 199.5 & 13.31 & 304.5 & 46.11 & $0.001208^{\circ}$ \\
\hline & \multirow{2}{*}{ Flexion } & Right (\%) & 112.0 & 11.13 & 157.2 & 21.40 & 0.003007 \\
\hline & & Left (\%) & 108.4 & 16.96 & 158.7 & 6.92 & $0.000278^{*}$ \\
\hline \multirow{4}{*}{ MRW } & \multirow{2}{*}{ Extension } & Right (J) & 129.6 & 44.25 & 289.2 & 49.70 & 0.000676 \\
\hline & & Left $(J)$ & 126.9 & 19.27 & 275.4 & 40.63 & $0.000077^{\circ}$ \\
\hline & \multirow{2}{*}{ Flexion } & Right (J) & 79.1 & 28.43 & 165.8 & 19.72 & $0.000511^{*}$ \\
\hline & & Left $(J)$ & 75.2 & 19.07 & 158.4 & 21.15 & $0.000182^{\prime}$ \\
\hline \multirow{4}{*}{ W/BW } & \multirow{2}{*}{ Extension } & Right (\%) & 197.3 & 70.28 & 238.2 & 105.04 & 0.490307 \\
\hline & & Left (\%) & 190.3 & 63.40 & 286.0 & 129.70 & 0.222222 \\
\hline & \multirow{2}{*}{ Flexion } & Right (\%) & 115.3 & 27.84 & 131.0 & 56.93 & 0.595640 \\
\hline & & Left (\%) & 106.8 & 14.09 & 133.1 & 28.65 & 0.103450 \\
\hline \multirow{4}{*}{ TW } & \multirow{2}{*}{ Extension } & Right $(J)^{* *}$ & $1,130.3$ & 512.10 & $2,128.7$ & $1,497.00$ & 0.690476 \\
\hline & & Left $(J)$ & 998.9 & 253.10 & 1612.2 & 588.82 & 0.064805 \\
\hline & \multirow{2}{*}{ Flexion } & Right (J) & 637.7 & 183.88 & 794.5 & 514.31 & 0.538825 \\
\hline & & Left $(\mathrm{J})$ & 582.6 & 107.37 & 870.7 & 262.30 & 0.052635 \\
\hline \multirow{4}{*}{ WF } & \multirow{2}{*}{ Extension } & Right $(\%)^{*+}$ & 2.5 & 6.90 & 13.4 & 90.90 & 0.841270 \\
\hline & & Left (\%) & -12.3 & 23.94 & -6.4 & 29.04 & 0.733277 \\
\hline & \multirow{2}{*}{ Flexion } & Right $(\%)^{* *}$ & 6.8 & 6.00 & 20.1 & 354.00 & 0.916815 \\
\hline & & Left (\%)" & -4.2 & 27.90 & 5.5 & 78.00 & 0.547619 \\
\hline \multirow{4}{*}{ AP } & \multirow{2}{*}{ Extension } & Right (W) & 165.2 & 52.90 & 213.8 & 124.03 & 0.444116 \\
\hline & & Left (W) & 164.3 & 35.86 & 238.6 & 95.22 & 0.140861 \\
\hline & \multirow{2}{*}{ Flexion } & Right (W) & 93.9 & 25.24 & 111.0 & 77.12 & 0.650833 \\
\hline & & Left (W) & 89.9 & 16.45 & 127.3 & 48.47 & 0.141528 \\
\hline & & Right (Nm) & 87.5 & 26.45 & 114.6 & 61.92 & 0.394733 \\
\hline TPT & Extension & Left (Nm) & 90.0 & 16.13 & 130.0 & 49.32 & 0.122874 \\
\hline API & & Right (Nm) & 50.9 & 8.90 & 62.7 & 38.42 & 0.521620 \\
\hline & Flexion & Left (Nm) & 52.9 & 5.76 & 72.4 & 23.23 & 0.105682 \\
\hline 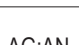 & & Right (\%)" & 53.7 & 4.60 & 58.6 & 2.10 & 0.420635 \\
\hline AG:AN & & Left (\%) & 56.0 & 13.09 & 58.9 & 8.93 & 0.687078 \\
\hline
\end{tabular}

"Difference between both groups is significant $(p<0.05)$.

"A nonparametric Mann-Whitney $U$ test was used. Results are median and the quartile gap.

Strength parameters from ankle joint testing are presented in Table 4. A greater number of the presented values were higher for the swimmers. Average MRW was about twice times significantly lower for gymnasts than swimmers. Significant differences were observed in W/BW values (higher in swimmers), non-significant only in W/ 
BW right ankle dorsi flexion. In plantar flexion TW was again significantly higher for swimmers in both sides. In dorsi flexion the significant difference was only noticed for the right side. Ankle WF parameter was higher in swimmers and the difference was significant only in dorsi flexion.

Tahle 4. Comparison of gymnasts and swimmers ankle strength parameters

\begin{tabular}{|c|c|c|c|c|c|c|c|}
\hline & & & \multirow{2}{*}{\multicolumn{2}{|c|}{ Gymnasts }} & \multirow{2}{*}{\multicolumn{2}{|c|}{ Swimmers }} & \multirow{3}{*}{ p } \\
\hline & & & & & & & \\
\hline & & & $\bar{x}$ & SD & $\bar{x}$ & SD & \\
\hline \multirow{4}{*}{ PT } & \multirow{2}{*}{ Plantar Flexion } & Right (Nm) & 71.5 & 20.37 & 113.8 & 46.89 & 0.101429 \\
\hline & & Left (Nm) & 70.9 & 12.76 & 105.6 & 35.65 & 0.074745 \\
\hline & \multirow{2}{*}{ Dorsi Flexion } & Right (Nm) & 25.4 & 3.75 & 28.0 & 4.29 & 0.341231 \\
\hline & & Left (Nm) & 25.0 & 5.88 & 26.4 & 3.40 & 0.643657 \\
\hline \multirow{4}{*}{ PT/BW } & \multirow{2}{*}{ Plantar Flexion } & Right (\%) & 110.3 & 27.00 & 147.2 & 57.66 & 0.231383 \\
\hline & & Left $(\%)$ & 110.5 & 20.63 & 136.7 & 43.56 & 0.258861 \\
\hline & \multirow{2}{*}{ Dorsi Flexion } & Right (\%) & 39.5 & 4.29 & 36.3 & 4.55 & 0.291262 \\
\hline & & Left (\%) & 38.6 & 7.66 & 34.2 & 3.09 & 0.269587 \\
\hline \multirow{4}{*}{ MRW } & \multirow{2}{*}{ Plantar Flexion } & Right (J) & 27.5 & 9.32 & 65.2 & 18.76 & $0.003831^{*}$ \\
\hline & & Left (J) & 26.0 & 4.72 & 63.0 & 20.40 & $0.004217^{*}$ \\
\hline & \multirow{2}{*}{ Dorsi Flexion } & Right (J) & 9.1 & 2.73 & 18.2 & 3.04 & $0.001066^{*}$ \\
\hline & & Left $(J)$ & 9.7 & 3.43 & 18.2 & 3.97 & $0.006838^{*}$ \\
\hline \multirow{4}{*}{ W/BW } & \multirow{2}{*}{ Plantar Flexion } & Right (\%) & 48.6 & 10.19 & 75.5 & 23.66 & $0.047663^{*}$ \\
\hline & & Left (\%) & 46.7 & 8.03 & 76.6 & 26.10 & $0.039956^{*}$ \\
\hline & \multirow{2}{*}{ Dorsi Flexion } & Right (\%) & 11.7 & 3.75 & 17.8 & 4.89 & 0.057863 \\
\hline & & Left $(\%)$ & 11.6 & 4.00 & 18.2 & 4.89 & $0.047187^{*}$ \\
\hline \multirow{4}{*}{ TW } & \multirow{2}{*}{ Plantar Flexion } & Right (J) & 271.9 & 63.80 & 493.5 & 135.72 & $0.010787^{*}$ \\
\hline & & Left $(J)$ & 235.2 & 59.89 & 479.0 & 179.44 & $0.020433^{*}$ \\
\hline & \multirow{2}{*}{ Dorsi Flexion } & Right (J) & 62.8 & 22.03 & 94.9 & 21.28 & $0.047369^{*}$ \\
\hline & & Left $(J)$ & 60.5 & 27.52 & 97.0 & 24.20 & 0.056561 \\
\hline \multirow{4}{*}{ WF } & \multirow{2}{*}{ Plantar Flexion } & Right (\%) & 1.4 & 18.10 & 10.3 & 15.51 & 0.424998 \\
\hline & & Left (\%) & 4.1 & 14.47 & 12.0 & 14.32 & 0.414117 \\
\hline & \multirow{2}{*}{ Dorsi Flexion } & Right (\%) & 32.0 & 13.29 & 52.4 & 8.51 & $0.020005^{*}$ \\
\hline & & Left (\%) & 31.9 & 9.47 & 52.6 & 4.90 & $0.002419^{*}$ \\
\hline \multirow{4}{*}{ AP } & \multirow{2}{*}{ Plantar Flexion } & Right (W) & 43.6 & 6.80 & 52.5 & 18.07 & 0.335871 \\
\hline & & Left (W) & 40.0 & 5.01 & 47.3 & 19.04 & 0.434622 \\
\hline & \multirow{2}{*}{ Dorsi Flexion } & Right (W) & 9.1 & 2.53 & 9.9 & 3.16 & 0.662408 \\
\hline & & Left (W) & 8.9 & 2.65 & 9.0 & 2.36 & 0.961066 \\
\hline & Plantar Flexion & Right (Nm) & 63.5 & 12.94 & 83.9 & 26.77 & 0.163909 \\
\hline APT & Plantar riexion & Left $(\mathrm{Nm})^{* *}$ & 55.5 & 2.50 & 80.3 & 29.90 & 0.309524 \\
\hline APT & Dorci Foyion & Right (Nm) & 16.5 & 3.00 & 16.8 & 3.91 & 0.902020 \\
\hline & Dorsi Flexion & Left (Nm) & 16.3 & 3.34 & 16.3 & 2.72 & 0.983922 \\
\hline$A G \cdot A N$ & & Right (\%) & 26.8 & 5.95 & 24.0 & 8.16 & 0.552489 \\
\hline AG:AIV & & Left $(\%)$ & 28.2 & 7.80 & 25.4 & 11.40 & 0.657907 \\
\hline
\end{tabular}

Difference between both groups is significant $(p<0.05)$.

"A nonparametric Mann-Whitney U test was used. Results are median and the quartile gap.

Table 5 presents the deficits of selected strength parameters for hip joints. The deficits of PT, MRW, TW and AP parameters between the hips, in both movements were lower in swimmers (the exception was a TW deficit in flexion), but a significant difference existed only for the MRW parameter during flexion. 
Table 5. Comparison of gymnasts and swimmers hip strength deficits between sides

\begin{tabular}{|c|c|c|c|c|c|c|c|}
\hline & & & \multirow{2}{*}{\multicolumn{2}{|c|}{ Gymnasts }} & & & \\
\hline & & & & & \multicolumn{2}{|c|}{ Swimmers } & \multirow{2}{*}{$p$} \\
\hline & & & $\bar{x}$ & SD & $\bar{x}$ & SD & \\
\hline \multirow{2}{*}{ PT } & Flexion & Deficit (\%) & 15.5 & 12.45 & 13.3 & 8.33 & 0.753188 \\
\hline & Extension & Deficit (\%) & 24.8 & 11.52 & 10.4 & 9.08 & 0.059386 \\
\hline \multirow{2}{*}{ MRW } & Flexion & Deficit (\%) & 33.8 & 14.74 & 13.4 & 7.91 & $0.026432^{\circ}$ \\
\hline & Extension & Deficit (\%) & 37.6 & 19.36 & 16.6 & 6.38 & 0.050213 \\
\hline \multirow{2}{*}{ TW } & Flexion & Deficit (\%) & 30.0 & 18.71 & 37.8 & 26.28 & 0.601624 \\
\hline & Extension & Deficit (\%) & 28.5 & 22.31 & 16.8 & 22.10 & 0.431191 \\
\hline \multirow{2}{*}{ AP } & Flexion & Deficit (\%) & 39.1 & 31.63 & 35.4 & 25.61 & 0.844807 \\
\hline & Extension & Deficit (\%) & 37.0 & 28.09 & 19.1 & 22.68 & 0.298796 \\
\hline
\end{tabular}

"Difference between both groups is significant $(p<0.05)$.

Comparison of strength parameter deficits for knee joints of gymnasts and swimmers is presented in Table 6. PT and MRW deficits were higher for gymnasts in both movements, with a significant difference only for MRW in extension. In contrast, almost all TW and AP deficits were higher for swimmers (except for the AP deficit in knee extension).

Table $\mathfrak{b}$. Comparison of gymnasts and swimmers knee strength deficits between sides

\begin{tabular}{|c|c|c|c|c|c|c|c|}
\hline & & & & & & & \\
\hline & & & \multicolumn{2}{|c|}{ Gymnasts } & \multicolumn{2}{|c|}{ Swimmers } & \multirow{2}{*}{$p$} \\
\hline & & & $\bar{x}$ & SD & $\bar{x}$ & $\mathrm{SD}$ & \\
\hline \multirow{2}{*}{ PT } & Extension & Deficit (\%) & 15.7 & 14.91 & 2.5 & 2.79 & 0.088308 \\
\hline & Flexion & Deficit (\%) & 14.2 & 5.38 & 10.5 & 3.86 & 0.244279 \\
\hline \multirow{2}{*}{ MRW } & Extension & Deficit (\%) & 20.5 & 12.80 & 1.0 & 1.50 & 0.031746 \\
\hline & Flexion & Deficit (\%) & 21.4 & 16.02 & 11.7 & 11.23 & 0.298863 \\
\hline \multirow{2}{*}{ TW } & Extension & Deficit (\%) & 15.4 & 11.58 & 29.8 & 38.47 & 0.446065 \\
\hline & Flexion & Deficit (\%) & 5.6 & 22.80 & 19.1 & 35.90 & 0.309524 \\
\hline \multirow{2}{*}{ AP } & Extension & Deficit (\%) & 20.7 & 7.80 & 13.1 & 23.00 & 0.690476 \\
\hline & Flexion & Deficit $(\%)^{* *}$ & 12.3 & 8.50 & 21.8 & 39.50 & 0.547619 \\
\hline
\end{tabular}

"Difference between both groups is significant $(p<0.05)$.

"A nonparametric Mann-Whitney $U$ test was used. Results are median and the quartile gap.

Table 7. Comparison of gymnasts and swimmers ankle strength deficits between sides

\begin{tabular}{|c|c|c|c|c|c|c|c|}
\hline & & & \multicolumn{2}{|c|}{ Gymnasts } & \multicolumn{2}{|c|}{ Swimmers } & \multirow[b]{2}{*}{$\mathrm{p}$} \\
\hline & & & $\bar{x}$ & SD & $\bar{x}$ & SD & \\
\hline \multirow{2}{*}{ PT } & Plantar Flexion & Deficit (\%) & 35.5 & 36.04 & 10.8 & 4.87 & 0.166995 \\
\hline & Dorsi Flexion & Deficit (\%) & 8.3 & 5.66 & 6.0 & 5.10 & 0.522022 \\
\hline \multirow{2}{*}{ MRW } & Plantar Flexion & Deficit (\%) & 29.3 & 27.67 & 18.7 & 13.47 & 0.463350 \\
\hline & Dorsi Flexion & Deficit (\%) & 10.9 & 9.61 & 12.3 & 11.59 & 0.838225 \\
\hline \multirow{2}{*}{ TW } & Plantar Flexion & Deficit (\%) & 23.4 & 18.15 & 20.2 & 16.03 & 0.772439 \\
\hline & Dorsi Flexion & Deficit (\%) & 11.1 & 11.79 & 14.7 & 10.19 & 0.613750 \\
\hline \multirow{2}{*}{ AP } & Plantar Flexion & Deficit (\%) & 13.6 & 12.01 & 11.6 & 6.15 & 0.756068 \\
\hline & Dorsi Flexion & Deficit (\%) & 6.0 & 3.21 & 8.8 & 5.83 & 0.367489 \\
\hline
\end{tabular}


Table 7 presents a comparison of deficits in selected strength parameters for the ankle joint. The differences between the deficits of all the presented strength parameters were lower for swimmers in the plantar flexion. However, in the movement of the dorsi flexion the results of swimmers were higher than gymnasts (except for the PT deficit). All described differences were not statistically significant for both movements.

\section{Discussion}

The results present values of selected strength parameters from highly qualified gymnasts and swimmers.

Hip joint PT of the gymnasts and swimmers were much higher than presented by Biodex (2019a) $13.4 \mathrm{Nm}$ in flexion and $21.0 \mathrm{Nm}$ in extension. The same was observed in hip PT/BW flexion (17.4\%) and extension (27.4\%). It is probably due to the fact that Biodex (2019a) presented the results of non-training subjects.

P.A. Garcia, J.M. Dias, R.C. Dias, P. Santos, C.C. Zampa (2011) presented hip joint AP parameters for 65-69, $70-79$ and $80+$ y.o. seniors. Both analyzed parameter sets were lower in the 65-69 and 70-79 y.o. seniors (83.6 $\pm 30.9 \mathrm{~W} ; 67.4 \pm 27.2 \mathrm{~W})$ in hip flexion AP. In hip extension movement the seniors AP (24.1-47.6 W) were much lower than gymnasts and swimmers. But it must be noticed that different angular velocities were used.

The APT for the dominant limb in hip flexion showed that gymnasts and swimmers were much lower than 65-69 and 70-79 y.o. seniors (74.7 $\pm 24.2 \mathrm{Nm} ; 63.1 \pm 24.0 \mathrm{Nm}$ ) (Garcia et al., 2011). APT values in gymnasts hip extension were just few percentages higher than 70-79 y.0. seniors (44.4 $\pm 23.2 \mathrm{Nm}$ ) (Garcia et al., 2011). Swimmers were more than 2 times higher than this. These unusual results are probably due to different angular velocities used in the measurement protocols.

Gymnasts presented almost four times higher values of AG:AN ratio than Biodex (2019a) (29\%). Swimmers also were higher than this, but as much as gymnasts. Greater balance of hip joint agonists and antagonists muscles can be seen in gymnasts than swimmers.

Results of these studies confirmed the thesis of T.D. Cahalan, M.E. Johnson, S. Liu, E.Y. Chao (1989). In their results hip extensors were stronger than flexors (regardless of age or gender). The same was in both researched groups.

Gymnast and swimmer PT and PT/BW measured in knee extension was greater than that showed by Biodex (2019a) (28.7 Nm; 38.4\%). Swimmer knee extension PT was almost the same as that presented by R. Dotan et al. (2013) for untrained men (22.9 y.o.) $-226.2 \pm 42.5 \mathrm{Nm}$, and at the same time, a bit higher than almost 19 y.o. male professional soccer players (Daneshjoo, Rahnama, Mokhtar, Yusof, 2013) - 201-209 Nm. Gymnasts were almost twice lower in the same strength parameter and movement. They archived values similar to older women (113-115 Nm) (Hakestad, Nordsletten, Torstveit, Risberg, 2014). This can be alarming. The same was observed in knee flexion PT. A. Daneshjoo et al. (2013) presented values of 100-102 Nm for young soccer players. It was higher for gymnasts and lower for swimmers, in this study.

In knee extension movement, TW parameter presented by gymnasts was lower than $50+$ y.o. women $(1,480-$ 1,536 J) (Hakestad et al., 2014). This can be caused by a lack of strength endurance training in this particular joint and movement. Swimmers results were much higher than this.

Both gymnasts and swimmers were much higher in knee AP in extension and flexion movements than seniors results (68.9-109.9 W; 23.8-43.2 W) (Garcia et al., 2011).

The APT parameter for investigated gymnasts and swimmers in knee extension was from more than 1.2 to almost 3 times higher than seniors (45.3-67.7 Nm) (Garcia et al., 2011). The same strength parameter in knee 
flexion were even more times higher than those presented by P.A. Garcia et al. (2011) (18.9-31.1 Nm). The APT parameter in knee flexion was from more than 1.5 to almost 4 times higher than seniors (18.9-31.1 Nm). Same strength parameter in knee extension was not so many times more than seniors (45.3-67.7 Nm) (Garcia et al., 2011) like above, but also higher in the investigated gymnasts and swimmers.

The knee AG:AN ratio presented by young male professional soccer players (Daneshjoo et al., 2013) was on average $50 \%$. Gymnasts were just a few percentage points higher and swimmers presented almost $9 \%$ more in the same parameter. Knee AG:AN results of non-training subjects (Biodex, 2019a) were much higher (72\%) than in this study, showing that professional sport can decrease the AG:AN knee ratio.

Measured ankle PT parameter in plantar flexion, for gymnasts were 4 to 5 times higher than showed by untrained subjects in Biodex (2019a) (16.4 Nm). But for swimmers it was almost 7 times more.

The same was observed in PT/BW values in the same movement for both groups in comparison to Biodex (2019a) data $-21.7 \%$. Ankle dorsi flexion PT values were about 6 times higher in gymnasts and swimmers than in untrained people $(4.3 \mathrm{Nm})$. In the same movement PT/BW values were 6-7 times higher than non-training people (5.7\%) (Biodex, 2019a).

B.A. Lee, S.H. Lee, D.J. Oh (2013) showed the results of the ankle joint for male university students representing modern pentathlon (21.00 \pm 1.15 y.o.). Ankle plantar flexion PT $(74.49 \pm 16.89 \mathrm{Nm})$ was just a few percentage points higher than gymnasts and much lower than swimmers. PT in ankle dorsi flexion $(\approx 20 \mathrm{Nm})$ was higher for both groups. But the results were measured in $60 \% \mathrm{sec}$ angular speed.

In ankle joint plantar flexion, both gymnasts and swimmers were almost 2 times lower in PT/BW parameter than gymnasts (182.45-189.99\%; 22.9 y.o.) and control group (185.84-191.01\%; 22.8 y.o.) presented by S.T. Aydog et al. (2005). PT/BW in dorsi flexion showed that gymnasts in our research were close to gymnasts investigated by S.T. Aydog et al. (2005) (36.79-46.53\%) but lower than controls (47.71-51.81\%). Swimmers were lower than both presented groups.

Gymnast and swimmer ankle APT in plantar flexion was higher than presented by seniors (24.9-47.2 Nm) (Garcia et al., 2011). The APT in dorsi flexion movement was higher than showed by 70-79 and 80+ y.o. (12.2-14.7 $\mathrm{Nm}$ ), but also almost the same as 65-69 y.o. people (16.8 $\pm 5.7 \mathrm{Nm}$ ) (Garcia et al., 2011).

The AG:AN ratios for ankle joints of gymnasts and swimmers were just few percentage points lower than showed by Biodex (2019a) - 31\% or S.T. Aydog et al. (2005) - 26.24-29.02\% in untrained people. Gymnasts in our study presented few percentages higher values than gymnasts in other research (Aydog et al., 2005) (23.34$25.03 \%$ ). This can prove that gymnastic and swimming training does not affect agonists to antagonists ratios in ankle joints.

All of the hip strength deficits were higher than acceptable (1-10\%) (Biodex, 2019b). Only swimmers PT deficits in flexion, in extension and MRW deficit in flexion movement were close to the considered limit. For gymnasts all of them were above.

According to Biodex (2019b) values (1-10\%), in knee joint, percentage differences between sides were acceptable only in flexion TW for gymnasts and extension PT or MRW in swimmers. Side deficits for gymnasts knee flexion AP and swimmers knee flexion PT, MRW and extension AP were near the proposed limit. In comparison, the PT deficits for young male professional soccer players (Daneshjoo et al., 2013) were higher in flexion movement (19.6\%) than both gymnasts and swimmers, but in extension (13.8\%) only swimmers showed lower values. 
In ankle joint dorsi flexion PT and AP deficits, both gymnasts and swimmers were within the recommended limit $\leq 10 \%$ (Biodex, 2019b). Other values, measured in the ankle joint, like MRW deficit, TW deficit in dorsi flexion or AP deficit in plantar flexion for gymnasts and PT deficit, AP deficit in plantar flexion or MRW deficit in dorsi flexion for swimmers were close but not within the limit of $10 \%$.

Measuring nine selected parameters, on both sides of the body (right and left lower limb), in two movements (flexion and extension or plantar and dorsi flexion - depending on measured joint) gave 34 strength parameters to compare between the examined groups for each tested joint.

In the hip joint we observed that 19 out of 34 parameters differed significantly between gymnasts and swimmers. Parameters were mostly related to the first "maximal strength" protocol. The analysis of knee joint presented 12 strength parameters, where differences between the examined groups were significant. They were related only to the first "maximal strength" protocol. Also in the ankle joint, there were 12 parameters that differed significantly between gymnasts and swimmers. In the majority they were related to the second "endurance-strength" protocol.

All of the results can give the conclusion that lower limb strength parameters differentiate highly qualified gymnasts and swimmers. The differences are probably caused by the specificity of the disciplines and the dissimilarity of training.

Analyzing differences between the gymnast and swimmer groups, the lowest $p$ value was observed in MRW flexion. For the knee joint the $p$ value was lowest in MRW in extension movement and PT in flexion movement. Also in the ankle joint, the MRW in both dorsi and plantar flexion had the lowest $p$ value. The MRW parameter was the one that differed gymnasts and swimmers the most, regardless of the examined joint.

Analyzing the strength parameter deficits, there were four for each joint, measured in both movements (flexion and extension or plantar and dorsi flexion - depending on the measured joint). That gave 8 deficits for each joint to analyze.

For hip strength deficits only MRW in hip flexion differed significantly between gymnasts and swimmers. In knee analysis, only in MRW deficit in extension movement the difference between groups was significant. None of the ankle joint strength deficits between sides were significant.

Only for hip and knee joints, 1 out of 8 deficits differed significantly. There was no significant difference between gymnast and swimmer deficits in ankle joints. This confirms the necessity of reaching bilateral development of the athlete's body (Alonso-Cortés Fradejas et al., 2006; Jurkojć et al., 2012; Gerhardt et al., 2014; Wiażewicz, 2015; Wiażewicz, 2016) in both, gymnastics and swimming, despite the discipline dissimilarity or training environment.

\section{Conclusions}

The obtained values of strength parameters for hip, knee and ankle joints from highly qualified athletes practicing gymnastics and swimming can be used in the training process, during control or selection, or to prevent injuries.

In order to achieve a high sport level, swimmers must demonstrate higher values of lower limbs strength parameters than gymnasts.

In the sport selection process in gymnastics and swimming, the value of work in the maximum repetition (MRW) of the lower limbs should be thoroughly analyzed. 
Training of highly qualified athletes practicing gymnastics or swimming should take into account the harmonious bilateral strength development of the lower limbs.

\section{Acknowledlgments}

No external financial support declared.

\section{References}

Alonso-Cortés Fradejas, B., Alvear-Órdenes, I., Ramírez-García, C., García-lsla, F.J., González-Gallego, J., Seco Calvo, J. (2006). Valoración isocinética del hombro en jóvenes nadadores mediante un patrón diagonal. Fisioterapia, 28 (6), 298-307. DOI: 10.1016/S0211-5638(06)74064-0.

Aydog, S.T., Ozçakar, L., Tetik, O., Demirel, H.A., Hasçelik, Z., Doral, M.N. (2005). Relation between foot arch index and ankle strength in elite gymnasts: a preliminary study. Br J Sports Med, 39 (3), e13. DOI: 10.1136/bjsm.2004.011627.

Bartkowiak, E. (1972). Pływanie sportowe. Warszawa: Sport i Turystyka.

Bartkowiak, E. (1984). Pływanie. Warszawa: Sport i Turystyka.

Biodex Medical Systems, Inc. (2019a). Biodex Multi-Joint System - Pro. Setup/Operation Manual. Retrieved from https://www.biodex. com/sites/default/files/850000man_08262revb.pdf.

Biodex Medical Systems, Inc. (2019b). Isokinetic Testing And Data Interpretation. Retrieved from https://www.biodex.com/sites/default/ files/manual-clinical-resources-data.pdf.

Bober, T., Hay, J.G. (1991). Dobór pozycji kątowych do pomiaru maksymalnych momentów sił mięśni w stawach kończyn człowieka. Zeszyty Naukowe AWF Wrocław, 53, 5-15.

Bogajewski, D., Roszko, R., Witkowski, M., Wróbel, W. (1969). Pływanie. Technika, metodyka i systematyka. Warszawa: AWF Warszawa.

Bołoban, W., Wiśniowski, W., Mistułowa, T., Niźnikowski, T. (2003). Metody aktywnego nauczania ćwiczeń akrobatycznych, złożonych koordynacyjnie. W: Międzynarodowa konferencja naukowa „Kierunki doskonalenia treningu i walki sportowej” (pp. 97-100). Warszawa: AWF Warszawa.

Cahalan, T.D., Johnson, M.E., Liu, S., Chao, E.Y. (1989). Quantitative measurements of hip strength in different age groups. Clin Orthop Relat Res, 246, 136-145.

Czabański, B., Fiłon, M., Zatoń, K. (eds) (2003). Elementy teorii pływania. Wrocław: AWF Wrocław.

Czajka, M., Wiśniowski, W., Sacewicz, T. (2016). The differences of legs strength possibilities of Artistic Gymnastics Elite athlete in two periods of sports preparation. VI Mjeżdunarodnoj Naućnoj Konfjerjencii Studjentow i Molodych Ućjenych: „Uniwjersitjeskij Sport: Zdorowje i Procwjetanie Nacii". Małachowka.

Daneshjoo, A., Rahnama, N., Mokhtar, A.H., Yusof, A. (2013). Bilateral and unilateral asymmetries of isokinetic strength and flexibility in male young professional soccer players. J Hum Kinet, 36 (1), 45-53. DOI: 10.2478/hukin-2013-0005.

Dotan, R., Mitchell, C., Cohen, R., Gabriel, D., Klentrou, P., Falk, B. (2013). Child-adult differences in the kinetics of torque development. J Sports Sci, 31 (9), 945-953. DOI: 10.1080/02640414.2012.757343.

Garcia, P.A., Dias, J.M., Dias, R.C., Santos, P., Zampa, C.C. (2011). A study on the relationship between muscle function, functional mobility and level of physical activity in community-dwelling elderly. Rev Bras Fisioter, 15 (1), 15-22. DOI: 10.1590/ S1413-35552011000100005.

Gerhardt, C., Doyscher, R., Boschert, H.P., Scheibel, M. (2014). The gymnastics shoulder. Orthopade, 43 (3) 230-235. DOI: 10.1007I s00132-013-2145-6.

Hakestad, K.A., Nordsletten, L., Torstveit, M.K., Risberg, M.A. (2014). Postmenopausal women with osteopenia and a healed wrist fracture have reduced physical function and quality of life compared to a matched, healthy control group with no fracture. BMC Womens Health, 14 (1), 92. DOI: 10.1186/1472-6874-14-92.

Jensen, R.H., Smith, G.L., Johnson, R.C. (1971). A technique for obtaining measurement of force generated by the hip muscle. Archives of Physical Medicine and Rehabilitation, 52 (5), 207-215.

Jurkojć, J., Michnik, R., Skubacz, H., Ziółkowska, E. (2012). Pomiary momentów sił mięśniowych w warunkach izokinetycznych u gimnastyków sportowych. Modelowanie Inżynierskie, 45 (14), 156-160. 
Kędzior, K., Niwiński, W., Wit, A. (1992). Pomiar momentów sił pojedynczych zespołów mięśniowych w warunkach dynamicznych. In: A. Wit (ed.), Biomechaniczna ocena układu ruchu sportowca (pp. 53-74). Warszawa: Instytut Sportu.

Laughlin, T. (2007). Pływanie dla każdego. Zielonka: Buk Rower.

Lee, B.A., Lee, S.H., Oh, D.J. (2013). Effects of peripheral injury in athletes with long-term-exercise participation in modern pentathlons. J Exerc Rehabil, 9 (5), 481-488. DOI: 10.12965/jer.130067.

Montgomery, J., Chambers, M. (2009). Mastering swimming. Champaign: Human Kinetics.

Przybylska, A. (2010). Pływanie kraulem. Warszawa: Landie.pl.

Sacewicz, T., Bodasiński, S., Śliwa, M., Szyszka, P., Czaplicki, A. (2016). Biomechanical assessment of motor abilities in male handball players during the annual training macrocycle. Polish Journal of Sport and Tourism, 23 (4), 190-195. DOI: 10.1515/pjst-2016-0025.

Sadowski, J., Bołoban, W., Mastalerz, A., Niźnikowski, T. (2003). Biomechaniczieskij analiz uzławych elementow sportiwnoj tiechniki akrobaticzieskich uprażnienij. VII Mieżdunarodnyj naucznyj kongres „Sowriemiennyj olimijskij sport i sport dla wsiech”. Tom II (pp. 274-275). Moskwa: RGUFK.

Sanders, R. (2013). How does asymmetries affect swimming performance? Journal of Swimming Research, 21, 1.

Troszczyński, J. (1999). Sportowa technika pływania. In: W. Iwanowski (ed.), Pływanie. Zagadnienia wybrane (pp. 81-105). Szczecin: Wydawnictwo Naukowe Uniwersytetu Szczecińskiego.

Wiażewicz, A. (2015). Izokinetyczna ocena parametrów siłowych u zawodników w Ośrodku Szkolenia Pływackiego w Szczecinie. In: J. Eider (ed.), Wybrane zagadnienia olimpijskie w teorii i praktyce (pp. 197-213). Szczecin: Wydawnictwo Naukowe Uniwersytetu Szczecińskiego.

Wiażewicz, A. (2016). Shoulder joint torque analysis in young swimmers. Antropomotoryka. Journal of Kinesiology and Exercise Sciences, 73 (26), 59-65. DOI: 10.5604/17310652.1226493.

Wit, A. (2002). Opracowanie biomechanicznej metody oceny stanu funkcjonalnego stawu biodrowego w procesie leczenia i rehabilitacji. Projekt badawczy. Warszawa: AWF.

Cite this article as: Wiażewicz, A., Wiśniowski, W., Sacewicz, T., Czajka, M. (2020). The Strength of Lower Limbs in Elite Gymnasts and Swimmers. Central European Journal of Sport Sciences and Medicine, 1 (29), 77-88. DOI: 10.18276/cej.2020.1-08. 